\title{
CLINICAL AND BIOMEDICAL APPLICATIONS OF SURFACE PLASMON RESONANCE SYSTEMS
}

\author{
David E. Meza-SÁnchez and José L. Maravillas-Montero*
}

Red de Apoyo a la Investigación, Universidad Nacional Autónoma de México (UNAM) and Instituto Nacional de Ciencias Médicas y Nutrición Salvador Zubirán, Mexico City, México

\begin{abstract}
Surface plasmon resonance (SPR)-based biosensors offer superior analytical features such as simplicity, sensitivity, and specificity when compared to conventional methods in clinical analyses. In addition, they deliver real-time monitoring of label-free analytes with high-throughput approaches requiring little sample pretreatment that allows the analysis of virtually every clinical sample type to determine the amount and/or activity of any molecule of interest. Accordingly, SPR emerges as a novel, efficient, powerful, and relatively low-cost alternative tool for routine clinical analysis, opening also new horizons for developments in personalized medicine applied to diagnostics or therapeutics' monitoring. (REV INVEST CLIN. 2019;71:85-90)
\end{abstract}

Key words: Surface plasmon resonance. Biomarker. Clinical diagnostics.

\section{INTRODUCTION}

Biosensors are analytical devices consisting of a biological sensing element connected to a physicochemical transducer. These elements generate digital electronic signals proportional to the interaction of biomolecules, thus allowing "label-free" detection of analytes. Due to the specificity of biomolecular interactions, a biosensor can be used to analyze complex substrates including samples such as blood, serum, plasma, urine, milk, and culture media often with minimum preparative treatment.

\section{Corresponding author:}

*José Luis Maravillas-Montero

Red de Apoyo a la Investigación

Instituto Nacional de Ciencias Médicas y Nutrición

Salvador Zubirán

Vasco de Quiroga, 15

Col. Sección XVI, Del. Tlalpan

C.P. 14080, Mexico City, Mexico

E-mail: maravillas@cic.unam.mx
Different types of biosensors have been developed including amperometric, potentiometric, piezoelectric, calorimetric, and optical biosensors. These have been applied mostly in food and water analysis and pharmaceutical processes due to their demands for sensitivity, specificity, speed, and accuracy of analyte measurements. Among them, optical biosensors correlating changes in concentration, mass, or number of molecules to direct changes in characteristics of light have preferentially evolved in these past years. Optical detection using surface plasmon resonance (SPR) biosensors has been increasingly popular due to its
Received for publication: 28-08-2018

Approved for publication: 25-09-2018

DOI: $10.24875 / R I C .18002754$ 
speed of detection, high specificity, high sensitivity, and possibility of real-time analysis ${ }^{1}$.

\section{SPR-BASED BIOSENSORS}

SPR appeared as a revolutionary technology $>20$ years ago, when their first commercial instrumentation, the Biacore, was launched on the market by the Swedish company Pharmacia Biosensors $A B^{2}$. Since then, many researchers adopted the SPR in various analytical fields such as the food industry, pharmaceutical approaches, doping analysis, proteomics and genomics, and environmental monitoring. Beyond that, clinical and biomedical analyses have also been explored with promising results ${ }^{1,3}$.

In a typical SPR experiment, one interacting molecule, referred as the "ligand," is bound to the biosensor surface while the other, called "analyte," is delivered to the surface in a continuous flow through a complex microfluidic system. The biosensor consists of a glass piece coated with a layer of gold, creating a platform for a range of specialized surfaces designed to optimize the binding of a variety of molecules including inorganic compounds, proteins, lipids, nucleic acids, carbohydrates, and even whole cells. The gold layer in the biosensor allows the generation of the SPR events that essentially detect changes in mass in the aqueous layer close to the biosensor surface by measuring changes in the refractive index of an incident polarized light beam (Fig. 1). The data obtained provide real-time quantitative information about binding specificity, active concentration of a molecule in a sample, kinetics, and affinity of binding models, among others. The complex theory behind SPR has been extensively detailed before ${ }^{4}$; thus, it will not be further discussed here.

\section{SPR APPROACHES FOR BIOMEDICAL AND CLINICAL APPLICATIONS}

SPR analyses possess a great potential for clinical and biomedical applications due to their inherent advantages when dealing with biomolecules. Optical SPR-based studies can be carried out with colored or even opaque samples, and there is no need to label molecules of interest with fluorescent or radioactive tags, thus avoiding the possibility that labels may compromise molecular activity or association features. In this way, analytes derived from clinical samples, human cells, or tissues can be studied in their native state to provide results that reflect more accurately their activity in vivo ${ }^{3,5}$.

In recent years, there has been a significant increase in research articles reporting the development of SPR techniques for biomolecular analysis; accordingly, several studies report the detection of different peptides, proteins (including antibodies), hormones, microRNA, and DNA. Consequently, multiple SPR-related reports emerged showing the analysis of clinical samples from patients suffering specific diseases, which is usually the ultimate objective of biomedical researchers looking for new diagnostic tools.

SPR approaches have been applied to clinical tests for monitoring antibodies, proteins, enzymes, drugs, small molecules, peptides, nucleic acids, and even bacterial components or viruses. Amazingly, while the molecules detected were usually at concentrations of nanomolar scales or higher, several studies reported picomolar detection or even lower concentrations. This technique has been applied in a number of biofluids including plasma, serum, whole blood, urine, stools, saliva, cervicovaginal secretion, endometrial tissue, synovial, cerebrospinal, amniotic, and ascites fluids ${ }^{1,3,6-9}$. The analytes detected by SPR in these samples represent an everyday growing list of molecules with great potential for clinical-related applications, as exemplified in the next sections and detailed in Table 1.

\section{Protein Biomarkers}

A protein biomarker is used in clinical diagnosis to monitor the status of several associated diseases, displaying a potential utility for targeted therapy and to evaluate the therapeutic responses. The implementation of proteomic approaches together with the growing number of clinical biomarkers of proteic nature is accelerating the development of reliable methods for their detection in complex clinical samples such as SPR techniques. Examples of these clinically relevant protein biomarkers include molecules related with specific diseases ${ }^{1,3,6-9}$, as explained below. 
Figure 1. Fundamentals of surface plasmon resonance (SPR) sensor. SPR is a physical phenomenon that occurs when polarized light strikes an electrically conducting surface (made of gold in most cases) at the interface between two media, a high-refractive-index glass or crystal prism and a low-refractive-index buffer solution. This generates intermittent waves of electrons (charge density waves), also known as plasmons, that reduce the intensity of reflected light at a specific angle known as the resonance angle (RA), in proportion to the mass deposited on the conducting surface. These arrays, referred to as SPR sensors, are used to detect a refractive index change of the polarized light beam within a detection area $(<500 \mathrm{~nm})$ as a change of the RA, when the interactions between ligand molecules (attached to the sensor) and the analytes (flowing in solution) occur.

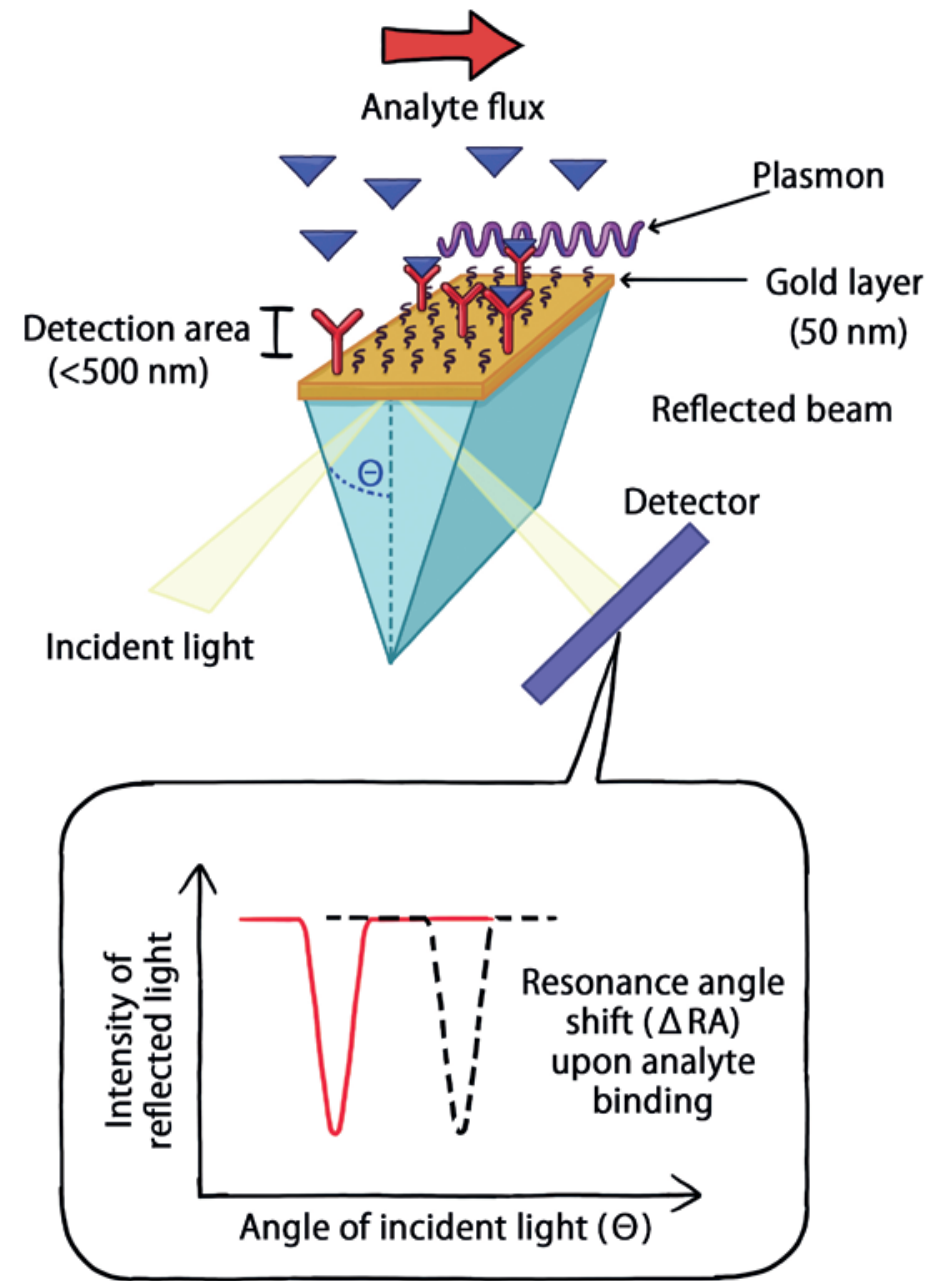

\section{Cancer Biomarkers}

An important aspect of all types of cancer management should include the monitoring of protein biomarkers related with these diseases, preferentially in easily collected physiological fluids over surgically obtained biopsies; examples of these proteins are the prostate-specific antigen, podoplanin, lipocalin 2, galectin 1, and CD166 (ALCAM), among others. Besides providing practical information to guide clinician's decisions, cancer biomarkers are also linked to specific alterations in molecular pathways controlling cancer pathogenesis, thus evidencing their potential for deciding about therapeutic strategies ${ }^{8,9}$.

\section{Cardiac Disease Biomarkers}

Cardiac biomarkers such as C-reactive protein, brain natriuretic peptide, myoglobin, and cardiac troponin I are proteins released into the bloodstream on damage of the heart or associated tissues. These biomarkers help to diagnose acute coronary syndrome 
Table 1 . Clinically relevant analytes determined by SPR analyses.

\begin{tabular}{|c|c|c|}
\hline Disease or condition & Analytes measured by SPR & Sample \\
\hline \multicolumn{3}{|l|}{ Antibodies } \\
\hline Antiphospholipid syndrome & Anti-cardiolipin, anti- $\beta 2$ glycoprotein I & Serum \\
\hline Chagas disease & Anti-Trypanosoma cruzi & Serum \\
\hline Dengue & Anti-dengue virus IgM antibodies & Serum \\
\hline Diabetes & Insulin and proinsulin autoantibodies & Serum \\
\hline Epstein-Barr virus infection & Anti-VCA, anti-EBNA, and anti-EA, all viral antigens & Serum \\
\hline Hepatitis A and B & Anti-hepatitis A or B antigens & Serum \\
\hline Leukemia & Anti-asparaginase, $\kappa$ and $\lambda$ immunoglobulin light chain & Serum \\
\hline Lyme disease & Anti-Borrelia spp. & Serum \\
\hline Neonatal thrombocytopenia & Anti-HPA-1a alloantibodies & Serum \\
\hline Peanut allergies & $\lg E$ & Serum \\
\hline Red cell aplasia & Anti-erythropoiesis-stimulating agent IgG4 antibodies & Serum \\
\hline Rheumatoid arthritis & $\begin{array}{l}\text { Anti-glucose } 6 \text {-phosphate isomerase, anti-citrullinated } \\
\text { protein antibodies }\end{array}$ & $\begin{array}{l}\text { Synovial fluid } \\
\text { and serum }\end{array}$ \\
\hline Syphilis & Anti-Treponema pallidum & Serum \\
\hline Systemic lupus erythematosus & Anti-dsDNA autoantibodies & Serum \\
\hline Typhoid fever & Anti-Salmonella enterica serotype typhi & Serum \\
\hline \multicolumn{3}{|l|}{ Other proteins } \\
\hline Alzheimer's disease & Tau protein & Serum \\
\hline Bladder cancer & Podoplanin & Serum, urine \\
\hline Cancer & Galectin-1 & Serum \\
\hline Cardiopulmonary bypass surgery & IL-2, IL-4, IL-6, IL-10, TNF- $\alpha$, and IFN- $\gamma$ & Serum \\
\hline Diabetes & Insulin & Serum \\
\hline Head and neck squamous cell carcinoma & 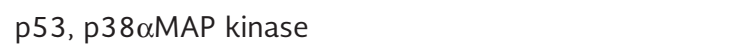 & Serum \\
\hline Heart disease & C-reactive protein, BNP, MG, and cTnl & Serum \\
\hline Hematopoiesis & $\mathrm{CXCL} 12$ & Urine \\
\hline Hepatocellular carcinoma & Lipocalin-2 & Serum \\
\hline Lung cancer & Rac & Serum \\
\hline Osteoarthritis & TNF- $\alpha$, MMP-3 & Synovial fluid \\
\hline Pancreatic cancer & ALCAM & Serum \\
\hline Preeclampsia & Albumin & Urine \\
\hline Prostate cancer & PSA & Serum \\
\hline Rheumatoid arthritis & Cathepsin G & Endometrial tissue \\
\hline Tuberculosis & CFP-10 & Urine \\
\hline \multicolumn{3}{|l|}{ Other molecules } \\
\hline Alzheimer's disease & Amyloid- $\beta$ & Cerebrospinal fluid \\
\hline Cancer & Exosomes & Ascites fluid \\
\hline Cancer treatment & Methotrexate & Serum \\
\hline Celiac disease & Gluten peptides & Urine \\
\hline Enterohemorrhagic Escherichia coli infection & PCR products of Escherichia coli 0157:H7 & Stool \\
\hline Fertility monitoring & Estriol 3-sulfate $16 \alpha$-glucuronide & Serum \\
\hline Pancreatic cancer & miR-21 and miR-10b & Plasma \\
\hline Sport doping & Human growth hormone & Serum \\
\hline$\beta$-thalassemia & Genomic DNA & Blood \\
\hline
\end{tabular}

Molecules used in clinical approaches, detected in human samples by SPR methods. The table was adapted from Mariani and Minunni ${ }^{3}$. PSA: prostate-specific antigen, SPR: surface plasmon resonance, IL: interleukin, Ig: immunoglobulin, TNF: tumor necrosis factor, IFN: interferon, BNP: brain natriuretic peptide, MG: myoglobin, cTnl: cardiac troponin I, PCR: polymerase chain reaction. 
and cardiac tissue ischemia, both conditions associated with insufficient blood flow. Cardiac biomarker proteins can also be used to estimate an individual's risk to develop these conditions or to help monitor and deal with a patient suspected to suffer from these conditions ${ }^{3}$.

\section{Antibodies}

Although SPR has been extensively applied for the characterization of monoclonal antibodies, both used in therapeutics or biomedical research, its utility in diagnosis by measuring antibody levels in circulation is a neglected application. However, there are reports of SPR playing a crucial role mainly in the analysis of autoantibodies in human serum for real-time monitoring of autoimmune disorders including for example anti-dsDNA autoantibodies in lupus erythematosus and anti-citrullinated protein antibodies in rheumatoid arthritis ${ }^{3}$.

Another field where antibody detection by SPR sensors could be important is the biopharmaceutical industry. Here, the immunogenicity of biological drugs is always a concern since they are primarily proteins (monoclonal antibodies, cytokines, growth factors, hormones, enzymes, or fusion proteins) and peptides that, when administered, often could induce a drugspecific immune response characterized by the presence of anti-drug antibodies (ADAs) ${ }^{10}$.

ADAs have been detected in clinical studies resulting in significant alterations in toxicology, pharmacokinetics, and efficacy of biotechnology-derived pharmaceuticals. Consequently, regulatory authorities are now requesting immunogenicity studies that include the detection of ADAs, before the approval of any biological drug ${ }^{10}$. On this scenario, the use of SPR devices for ADAs detection represents a valuable approach due to the intrinsic and previously discussed advantages of this technology.

\section{Hormones Measurement}

The analysis of both lipidic and small peptide hormones is an important area in clinical diagnostics and, currently, in anti-doping regulations. Endocrine diseases, where these mediators are typically involved, usually require measuring hormone levels by direct or indirect methods. Accordingly, SPR has demonstrated the ability to provide sensitive solutions in this clinical area by determining, for example, estriol metabolites and human growth hormone, among other hormones ${ }^{3,7}$.

\section{Nucleic Acid Analyses}

SPR assays related with nucleic acids could be subdivided into two main classes: analyses for the detection of chromosome abnormalities including point mutations and single-nucleotide polymorphisms such as those occurring in $\beta$-thalassemia, and assays for the quantification of genetic material as biomarkers such as microRNAs ${ }^{3}$.

\section{Pathogens Detection}

Beyond the identification of pathogens through specific DNA sequences targeting, other approaches based on the detection of a number of cell pathogen components have been reported. Particularly, several bacterial and parasite components, including Salmonella spp. and Schistosoma mansoni antigens, or even whole of H1N1 influenza virus particles, have been subject to SPR analyses ${ }^{6}$.

\section{Whole-cell SPR Analyses}

The detection depth of conventional SPR sensors reaches no $>500 \mathrm{~nm}$ beyond the gold layer; therefore, they are useful for the detection of changes near the plasma membrane of live cells with a high sensitivity. In this way, different SPR techniques have been developed to perform real-time evaluation of exogenous stimuli-induced responses in living cells. These approaches might reflect the reorganization of proteins as a consequence of intracellular signal transduction processes.

Examples of whole-cell analyses by SPR devices include the detection of real-time adhesion and morphological changes in living cells due to the action of toxins or enzymes ${ }^{5}$. A similar approach is the SPR measurement of the apoptosis rate of cancer cells in response to different oncological drugs, which can be applied in a clinical setting to evaluate the individual therapeutic potential of different treatments including pharmacodynamic interactions ${ }^{5}$. In addition, SPR sensors could reveal real-time alterations in intracellular signaling pathways of abnormal cells such as 
cancer cells, making them able to detect malignant tumors 5 .

Finally, the recently developed SPR imaging systems and the long-range SPR sensors for living cells may allow the visualization and deep through analyses of single cell reactions, potentially expanding the application of SPR whole-cell sensing for clinical diagnosis in the near future.

\section{CONCLUSIONS}

To be implemented as a routine analytical method, SPR sensing will need to replace existing technologies. In this way, several clinical results obtained with SPR devices have been compared to those from ELISA assays, chemiluminescence approaches, polymerase chain reaction, or liquid chromatography-mass spectrometry, generally demonstrating similar quality ${ }^{8}$.

Since ELISA employs the same experimental design as SPR sensing, regarding the detection of analytes using direct, indirect, or sandwich assays, it is frequently the method against which SPR systems are compared. The studies show that ELISA and SPR deliver the same dynamic range of sensitivity, although the advantages of SPR reside in the label-free and rapid detection of analytes plus the possibility of reusing the same sensor many times, which could decrease the cost of serial measurements. The SPR method is also preferred for the detection of molecules with low binding affinities, which can otherwise be washed away in ELISA assays.

Although the detection of antibody and general protein markers dominates the current applications of SPR approaches that undoubtedly support medical practice, the increasingly recognized usefulness of genetic testing, microRNA detection, and special molecules' analyses in clinical practice could represent an interesting niche for SPR applications.
In the upcoming years, novel integrated, simpler, and even portable SPR systems, capable of detecting biomolecules with high sensitivity ideally in undiluted biofluids, will be surely available to clinicians for daily use, allowing for a more accurate decision-making in the management of a given patient. For now, the promising results of this technology for the analysis of human samples in detecting biomarkers and helping with diagnostic or prognostic approaches in several diseases position SPR techniques as a powerful tool in clinical management with a great potential in precision medicine.

\section{ACKNOWLEDGMENTS}

This work was supported by grants 240314 from CONACYT as well as IA204316 and IA202318 from UNAM-DGAPA-PAPIIT program. The authors thank Ari Kleinberg-Bild (RAI, UNAM) for the art work of Figure 1.

\section{REFERENCES}

1. Rich RL, Myszka DG. Advances in surface plasmon resonance biosensor analysis. Curr Opin Biotechnol. 2000;11:54-61.

2. Malmqvist M. BIACORE: an affinity biosensor system for characterization of biomolecular interactions. Biochem Soc Trans. 1999;27:335-40

3. Mariani S, Minunni M. Surface plasmon resonance applications in clinical analysis. Anal Bioanal Chem. 2014;406:2303-23.

4. Wang DS, Fan SK. Microfluidic surface plasmon resonance sensors: from principles to point-of-care applications. Sensors (Basel). 2016;16:E1175

5. Yanase $Y$, Hiragun $T$, Ishii $K$, et al. Surface plasmon resonance for cell-based clinical diagnosis. Sensors (Basel). 2014;14:4948-59.

6. Bergwerff AA, van Knapen F. Surface plasmon resonance biosensors for detection of pathogenic microorganisms: strategies to secure food and environmental safety. J AOAC Int. 2006;89: 826-31.

7. Wittenberg NJ, Wootla B, Jordan LR, et al. Applications of SPR for the characterization of molecules important in the pathogenesis and treatment of neurodegenerative diseases. Expert Rev Neurother. 2014;14:449-63.

8. Masson JF. Surface plasmon resonance clinical biosensors for medical diagnostics. ACS Sens. 2017;2:16-30.

9. Ferhan AR, Jackman JA, Park JH, Cho NJ, Kim DH. Nanoplasmonic sensors for detecting circulating cancer biomarkers. Adv Drug Deliv Rev. 2018;125:48-77.

10. Gunn GR 3rd, Sealey DC, Jamali F, et al. From the bench to clinical practice: understanding the challenges and uncertainties in immunogenicity testing for biopharmaceuticals. Clin Exp Immunol. 2016;184:137-46. 